\title{
A Time Dependent Born-Oppenheimer Approximation
}

\author{
George A. Hagedorn`

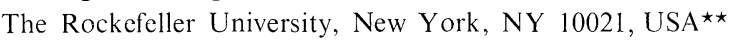

\begin{abstract}
We consider the dynamics of a quantum mechanical system which consists of some particles with large masses and some particles with small masses. As we increase the large masses to infinity we obtain the following results: The particles of smaller mass move adiabatically and determine an effective potential in which the heavier particles move semiclassically. Our methods can be applied to diatomic molecules with Coulomb forces.
\end{abstract}

\section{Introduction}

In 1927 Born and Oppenheimer [2] studied the bound state energies of molecular quantum systems. These systems were characterized by having two types of particles: the nuclei with large masses on the order of $M$, and the electrons with smaller masses on the order of $m$. The authors argued that the energy levels had an asymptotic expansion in the parameter $(m / M)^{1 / 4}$, and gave physical interpretations for the non-zero terms up to fourth order.

Although the Born-Oppenheimer approximation has been very useful for understanding molecules, there has been little mathematical work concerning its validity. However, two announcements [3,4] of rigorous results by Aventini, Combes, Duclos, Grossman and Seiler have been published.

Rather than study the bound state energies of such systems, we will study the evolution of certain states as the large mass $M$ tends to infinity. In order to obtain a non-trivial limit, we will take the velocities of the heavier particles to be on the order of $M^{-1 / 2}$ and study the motion for times on the order of $M^{1 / 2}$. Our results show that as we take $M$ to infinity, the lighter particles move adiabatically and determine an effective potential in which the heavier particles move semiclassically.

This time dependent approximation is widely used in the chemical physics literature, where it is usually attributed to Born and Oppenheimer [2]. However, no time dependent problems are considered in [2], and we believe the idea for the approximation was originally suggested by London in [14]. For a review of the subject as of 1935 , see [6].

* Supported in part by the National Science Foundation under Grant PHY 78-08066

$\star \star$ Present address: Department of Mathematıcs, Virginıa Polytechnic Institute and State University, Blacksburg, VA 24060 USA 
The paper is organized as follows. In Sect. 2 we establish notation, make several remarks, and precisely state our theorem. A heuristic discussion of the proof of this theorem is given in Sect. 3. The details of the proof appear in Sect. 4.

\section{Description of the Results}

The main purpose of this section is to state our theorem, but it is necessary for us to begin with a several definitions, remarks and formulas.

We consider a system of $n$ particles moving in $m$ dimensions. The mass of the $j$ th particle is $\beta^{-2} M_{j}$ for $1 \leqq j \leqq k$ and $m_{j}$ for $k+1 \leqq j \leqq n$. The position of the $j$ th particle is $x_{j}$, and $\Delta_{j}$ denotes the Laplacian with respect of $x_{j}$. The Hamiltonian for this system is given by

$$
H(\beta)=-\sum_{j=1}^{k}\left(2 M_{j}\right)^{-1} \beta^{2} \Delta_{j}-\sum_{j=k+1}^{n}\left(2 m_{j}\right)^{-1} \Delta_{j}+\sum_{i<j} V_{i j}\left(x_{i}-x_{j}\right) \text { on } \mathscr{H}=L^{2}\left(\mathbb{R}^{n m}\right)
$$

For all $i$ and $j$ we assume $V_{i j} \in L^{p}\left(\mathbb{R}^{m}\right)+L^{\infty}\left(\mathbb{R}^{m}\right)$ for some $p>m / 2$ if $m \geqq 3, p>1$ if $m=2$, and $p=1$ if $m=1$, so that $H(\beta)$ is self-adjoint. For $1 \leqq i<j \leqq k$ we assume $V_{i j}$ is twice continuously differentiable with second derivative uniformly Lipshitz on compact subsets of $\mathbb{R}^{m}$. For $1 \leqq i \leqq k<j \leqq n$ we take $V_{i j}$ to be real analytic.

\section{Remarks}

1. We will be interested in the $\beta \rightarrow 0$ limit of the dynamics generated by $H(\beta)$.

2. The conditions we have placed on $V_{i j}$ for $1 \leqq i<j \leqq k$ are stronger than necessary. Our arguments can easily be modified to allow these potentials to be repulsive Coulomb potentials.

3. The analyticity condition on $V_{i j}$ for $1 \leqq i \leqq k<j \leqq n$ is also stronger than necessary. With simple alterations, our methods can accommodate cases in which these potentials are twice continuously differentiable with second derivatives uniformly Lipshitz on compact sets.

4. Our methods can also be extended to accommodate diatomic molecules and ions (in which all the potentials are Coulombic). Combes and Seiler [5] have shown that the electron energies of these systems are analytic functions of the nuclear separation for nonzero separation, and that the corresponding electron wave functions are $C^{2}$ functions of the separation. This is sufficient information for the extension of our proof to diatomic Coulomb systems. However, we presently do not know how to handle molecules or ions which contain more that two nuclei.

For convenience we set $R=\left(x_{1}, x_{2}, \ldots, x_{k}\right)$ and $r=\left(x_{k+1}, x_{k+2}, \ldots, x_{n}\right)$. We denote the gradient of a function $f$ with respect to $R$ by $f^{(1)}(R)$, and let $\left|f^{(1)}(R)\right|$ denote its Euclidean norm. The symbol $f^{(2)}(R)$ denotes the Hessian $\frac{\partial^{2} f}{\partial R_{i} \partial R_{j}}(R)$, and $\left\|f^{(2)}(R)\right\|$ denotes its norm as an operator on $\mathbb{C}^{(k-1) m}$.

The Hamiltonian $H(\beta)$ can be written as

$H(\beta)=X(\beta)+h+W$. where 


$$
\begin{aligned}
& X(\beta)=-\sum_{j=1}^{h-1}\left(2 M_{j}\right)^{-1} \beta^{2} \Delta_{j}, \\
& h=-\sum_{j=k+1}^{n}\left(2 m_{j}\right)^{-1} \Delta_{j}+\sum_{\substack{k<j \\
i<j}} V_{i j}\left(x_{i}-x_{j}\right),
\end{aligned}
$$

and $W=\sum_{i<j \leqq k} V_{i j}(R)$.

For convenience we assume $M_{1}=M_{2}=\ldots=M_{k}=1$.

The operator $h(\beta)$ on $\mathscr{H}=L^{2}\left(\mathbb{R}^{n m}\right)$ is decomposed by the direct integral decomposition $\mathscr{H}=\int_{\mathbb{R} k m} \oplus L^{2}\left(\mathbb{R}^{(n-k) m}, d r\right) d R$. In other words, for each $R \in \mathbb{R}^{k m}$ there is an operator $h(R)$ on $L^{2}\left(\mathbb{R}^{(n-k) m}, d r\right)$ such that the action of $h$ on the function $f(R, r) \in \mathscr{H}$ is given by $[h f](R, r)=[h(R) f(R, \cdot)](r)$. The operators $h(R)$ on the fibers are

$$
h(R)=-\sum_{j=k+1}^{n}\left(2 M_{j}\right)^{-1} \Delta_{j}+\sum_{\substack{k<j \\ i<j}} V_{i j}\left(R, x_{k+1}, x_{k+2}, \ldots, x_{n}\right) .
$$

We assume the existence of an open set $U \subseteq \mathbb{R}^{k m}$ such that $h(R)$ has a nondegenerate isolated eigenvalue $E(R)$ whenever $R \in U$. We let $P(R)$ denote the spectral projection for $h(R)$ corresponding to $E(R)$. Note that since we have not removed the center of mass motion of the system, we can always extend $U$ to be of the form $U=\mathbb{R}^{m} \times \tilde{U}$ in a suitable coordinate system.

For $R \in U$ we let $\phi(R, r)$ be the normalized eigenfunction of $h(R)$ corresponding to $E(R)$. By our hypotheses on the potentials we can choose $\phi(R, r)$ to be real and continuous in $R$. This determines $\phi(R, r)$ up to $a \pm$ sign, which we arbitrarily choose.

For $R \notin U$ we define $E(R)=0$ and $\phi(R, r)=0$.

As mentioned in the introduction, our goal is to study the small $\beta$ behavior of certain solutions of $i \frac{\partial \psi}{\partial s}=H(\beta) \psi$ for times $s$ on the order of $\beta^{-1}$ and with the initial velocities of the heavy particles on the order of $\beta$. For convenience we replace $s$ by $t=\beta$ S and study $i \beta \frac{\partial \psi}{\partial t}=H(\beta) \psi$ for $t$ in a fixed interval $[0, T]$ and with fixed initial heavy particle velocities (with respect to $t$ ).

In the $t$ variables the $\beta \rightarrow 0$ limit looks formally like the $\hbar \rightarrow 0$ limit for the heavy particles. So, we will make use of some semiclassical techniques. In particular, we will use the tools developed in $[7,8,9]$ (see also $[10,11]$ ) to study the motion of the heavy particles in certain Gaussian states $\psi_{\alpha}(A, B, \beta, a, \eta, R)$. They are defined as follows:

Definition. Let $A$ and $B$ be complex $\mathrm{km} \times \mathrm{km}$ matrices which satisfy the following conditions:

$A$ and $B$ are invertible;

$B A^{-1}$ is symmetric ([real symmetric $]+i$ [real symmetric $\left.]\right)$; 
$\operatorname{Re} B A^{-1}=\frac{1}{2}\left[\left(B A^{-1}\right)+\left(B A^{-1}\right)^{*}\right]$ is strictly positive definite;

$\left(\operatorname{Re} B A^{-1}\right)^{-1}=A A^{*}$.

For all $a \in \mathbb{R}^{k m}, \eta \in \mathbb{R}^{k m}, \alpha \in[0,1]$, and $\beta>0$, we define

$$
\begin{aligned}
\psi_{\alpha}(A, B, \beta, a, \eta, R)= & (2 \pi)^{-k m / 4} \beta^{-k m \alpha / 2}(\operatorname{det} A)^{-1 / 2} \\
& \cdot \exp \left\{-\left(4 \beta^{2 \alpha}\right)^{-1}\left\langle(R-a), B A^{-1}(R-a)\right\rangle+i\langle\eta,(R-a)\rangle / \beta\right\} .
\end{aligned}
$$

The choice of the square root in this definition will depend on the context and will always be specified.

\section{Remarks}

1. If $A$ and $B$ satisfy conditions (2.1)-(2.4), then these conditions are also satisfied when $A$ and $B$ are interchanged. This is due to the fact that conditions (2.1) and (2.4) are equivalent to $A^{*} B+B^{*} A=2 I$ (see the proof of Lemma 2.1 of [8]). This condition is clearly invariant under interchange of $A$ and $B$.

2. Whenever we write $\psi_{\alpha}(A, B, \beta, a, \eta, R)$, we tacitly assume conditions (2.1)(2.4) are satisfied. Consequently, we will always be dealing with normalized heavy particle states.

3. The state $\psi_{\alpha}(A, B, \beta, a, \eta, R)$ is concentrated near the position $a$. Its position uncertainty is given by the matrix $\beta^{\alpha}\left(A A^{*}\right)^{1 / 2}$. The velocity is concentrated near $\eta$, with velocity width matrix $\beta^{1-\alpha}\left(B B^{*}\right)^{1 / 2} / 2$. Note that due to our scaling of masses and time, the velocity operator is $-i \beta \nabla_{R}$.

As well as being a semiclassical limit for the heavy particles, the $\beta \rightarrow 0$ limit is an adiabatic limit for the lighter particles. However, the situation is complicated by the coupling between the heavy particle motion and the light particle motion. At $t=0$ we take the state of the full system to be

$$
\psi_{\alpha}(A(0), B(0), \beta, a(0), \eta(0), R) \phi(R, r),
$$

where $a(0) \in U$. The adiabatic approximation predicts that the state of the lighter particles at some later time $t \neq 0$ will approximately be

$$
e^{-i t h(R) / \beta} \phi(R, r)=e^{-i t E(R) / \beta} \phi(R, r) .
$$

It is reasonable to expect the rapidly oscillating factor $e^{-i t E(R) / \beta}$ to affect the heavy particle motion so that the heavy particle state at time $t$ is approximately equal to

$$
e^{-i t(X(\beta)+W(R)+E(R)) / \beta} \psi_{\alpha}(A(0), B(0), \beta, a(0), \eta(0), R) .
$$

The semiclassical approximation $[7,8,9]$ shows that for small $\beta$, this heavy particle state approximately equals

$$
e^{i S(t) / \beta} \psi_{\alpha}(A(t), B(t), \beta, a(t), \eta(t), R),
$$

where $S(t), A(t), B(t), a(t)$, and $\eta(t)$ satisfy the following system of ordinary differential equations:

$$
\frac{d a}{d t}(t)=\eta(t)
$$




$$
\begin{aligned}
& \frac{d \eta}{d t}(t)=-E^{(1)}(a(t))-W^{(1)}(a(t)), \\
& \frac{d S}{d t}(t)=[\eta(t)]^{2} / 2-E(a(t))-W(a(t)), \\
& \frac{d A}{d t}(t)=i \beta^{1-2 \alpha} B(t) / 2, \\
& \frac{d B}{d t}(t)=2 i \beta^{2 \alpha-1}\left[E^{(2)}(a(t))+W^{(2)}(a(t))\right] A(t) .
\end{aligned}
$$

Our theorem shows that the heuristic picture we have just presented is more or less correct. So, we will require some information about solutions to (2.6)-(2.9). Given $a_{0} \in U$ and $\eta_{0} \in \mathbb{R}^{k m}$, standard techniques [1] show the existence of $T>0$ such that there is a unique solution $[a(t), \eta(t), S(t)]$ to $(2.5)-(2.7)$ on $\{t: t \in[0, T]\}$ which satisfies $a(0)=a_{0}, \eta(0)=\eta_{0}, S(0)=0$, and $a(t) \in U$. Furthermore, given $A_{0}$ and $B_{0}$, there exists a unique solution $[A(t), B(t)]$ to $(2.8)-(2.9)$ on $\{\mathrm{t}: t \in[0, T]\}$ such that $A(0)=A_{0}$ and $B(0)=B_{0} . A(t)$ and $B(t)$ satisfy conditions (2.1)-(2.4) if these conditions are satisfied by $A_{0}$ and $B_{0}$ (see Lemma 2.1 of [8]). Our hypothesis also guarantee the existence of the partial derivatives $\frac{\partial a(t)}{\partial a(0)}, \frac{\partial a(t)}{\partial \eta(0)}, \frac{\partial \eta(t)}{\partial a(0)}, \frac{\partial \eta(t)}{\partial \eta(0)}$, and the following relations are valid $[8$, Sect. 2$]$ :

$$
\begin{aligned}
& A(t)=\frac{\partial a(t)}{\partial a(0)} A(0)+\frac{1}{2} i \beta^{1-2 \alpha} \frac{\partial a(t)}{\partial \eta(0)} B(0), \\
& B(t)=\frac{\partial \eta(t)}{\partial \eta(0)} B(0)-2 i \beta^{2 \alpha-1} \frac{\partial \eta(t)}{\partial a(0)} A(0) .
\end{aligned}
$$

Under the circumstances described above we can draw the following conclusions.

Theorem 2.1. Let $H(\beta), E(R), \phi(R, r), T$, etc. be as above. Then given $\alpha \in(1 / 3,1 / 2]$, there exist $C$ and $b>0$ such that $0<\beta \leqq b$ implies

$$
\begin{aligned}
& \| e^{-i t H(\beta) / \beta} \psi_{\alpha}(A(0), B(0), \beta, a(0), \eta(0), R) \phi(R, r) \\
& -e^{i S(t) / \beta} \psi_{\alpha}(A(t), B(t), \beta, a(t), \eta(t), R) \phi(R, r) \| \leqq C \beta^{3 \alpha-1}
\end{aligned}
$$

for all $t \in[0, T]$.

Corollary 2.2. Theorem 2.1 remains true if (2.12) is replaced by

$$
\begin{aligned}
& \| e^{-i t H(\beta) / \beta} \psi_{\alpha}(A(0), B(0), \beta, a(0), \eta(0), R) \phi(a(0), r) \\
& -e^{i S(t) / \beta} \psi_{\alpha}(A(t), B(t), \beta, a(t), \eta(t), R) \phi(a(t), r) \| \\
& \quad \leqq C \beta^{3 \alpha-1}
\end{aligned}
$$

\section{Remarks}

1. Both the theorem and the corollary show that as $\beta \rightarrow 0$, the lighter particles 
move adiabatically and determine an effective potential in which the heavier particles move semiclassically.

2. Inequalities (2.12) and (2.13) imply one another (except that the $C$ 's may be different). This follows from the fact that given any $A, B, a \in U$, and $\eta$, there exists $c>0$ so that

$$
\left\|\psi_{\alpha}(A, B, \beta, a, \eta, R)[\phi(R, r)-\phi(a, r)]\right\| \leqq c \beta^{3 \alpha-1} .
$$

This inequality may be established by mimicking the proof of Lemma 4.1 below.

3. The vectors involved in (2.13) are normalized. Unless $\mathbb{R}^{k m} \backslash U$ has Lebesgue measure zero, the vectors in (2.12) are not normalized since $\phi(R, r)=0$ for $R \notin U$. Also, (2.13) is more reminiscent of the Born-Oppenheimer approximation since the variables $R$ and $r$ have been completely separated.

4. Theorem 2.1 and Corollary 2.2 both trivially extend to accommodate identical particles, but one should keep in mind the non-degeneracy of the energy $E(R)$ for $R \in U$.

\section{Main Ideas of the Proof}

In this section we present a heuristic discussion of the proof of Theorem 2.1 since the detailed proof involves several technical lemmas which obscure the principal ideas. Part A of the section contains an outline of the proof under the assumption that a certain lighter particle vector $\phi_{1}(R, \beta \eta, \cdot) \in L^{2}\left(\mathbb{R}^{(n-k) m}, d r\right)$ can be chosen. Part B indicates how that choice can be made.

\section{A. Outline of the Proof}

Theorem 2.1 shows that up to a possible phase factor, the lighter particles can approximately be found in the state $\phi(R, r)$ at any time $t \in[0, T]$ if they were in that state at $t=0$. This result is a zeroth order adiabatic approximation, and a first order approximation is required for our proof. For this reason we must introduce a vector $\phi_{1}\left(R, \beta \eta,^{\cdot}\right)$.

The vector $\phi_{1}$ will be chosen to have the following properties:

$$
\begin{aligned}
& \left\|e^{-i s h(R) / \beta} \phi_{1}(R, \beta \eta, \cdot)-e^{-i s E(R) / \beta} \phi_{1}(R+s \eta, \beta \eta, \cdot)\right\|_{L^{2}(d r)} \\
& \quad \leqq C|s| \beta^{3 \alpha-1}
\end{aligned}
$$

for all $\eta$ in any given compact set and all sufficiently small $s$;

$$
\left\|\phi_{1}(R, \beta \eta, \cdot)-\phi_{1}\left(R, \beta \eta^{\prime} \cdot \cdot\right)\right\|_{L^{2}(d r)} \leqq C \beta\left|\eta-\eta^{\prime}\right|
$$

for all $\eta$ and $\eta^{\prime}$ in any given compact set containing $\eta=0$; and

$$
\phi_{1}(R, 0, r)=\phi(R, r) \text {. }
$$

Assuming the existence of $\phi_{1}$ (see Part B), we begin our proof by using (3.2) and (3.3) to approximate

$$
\begin{aligned}
& e^{-i t H(\beta) / \beta} \psi_{\alpha}(A(0), B(0), \beta, a(0), \eta(0), R) \phi(R, r) \quad \text { by } \\
& e^{-i t H(\beta) / \beta} \psi_{\alpha}(A(0), B(0), \beta, a(0), \eta(0), R) \phi_{1}(R, \beta \eta(0), r) .
\end{aligned}
$$


Next we observe that $e^{-i s[h+W] / \beta}=e^{-i s W / \beta} e^{-i s h / \beta}$ since $h$ commutes with multiplication by functions of $R$. So, the Trotter Product Formula [16] allows us to approximate

$$
\begin{aligned}
& e^{-i t H(\beta) / \beta} \psi_{\alpha}(A(0), B(0), \beta, a(0), \eta(0), R) \phi_{1}(R, \beta \eta(0), r) \text { by } \\
& \left\{e^{-i t X(\beta) / \beta N} e^{-i t W / \beta N} e^{-i t h / \beta N}\right\}^{N} \\
& \quad \cdot \psi_{\alpha}(A(0), B(0), \beta, a(0), \eta(0), R) \phi_{1}(R, \beta \eta(0), r)
\end{aligned}
$$

for sufficiently large $N$.

We now concentrate on the action of the first of the $N$ factors in the product. Inequality (3.1) shows that up to an error on the order of $t \beta^{3 \alpha-1} / N$,

$$
e^{-i t X(\beta) / \beta N} e^{-i t W / \beta N} e^{-i t h / \beta N} \psi_{\alpha}(A(0), B(0), \beta, a(0), \eta(0), R) \phi_{1}(R, \beta \eta(0), r)
$$

can be approximated by

$$
\begin{aligned}
& e^{-i t X(\beta) / \beta N} e^{-i t[W(R)+E(R)] / \beta N} \\
& \quad \cdot \psi_{\alpha}(A(0), B(0), \beta, a(0), \eta(0), R) \phi_{1}(R+t \eta(0) / N, \beta \eta(0), r) .
\end{aligned}
$$

In this expression we replace $W(R)+E(R)$ by its second order Taylor expansion

$$
\begin{gathered}
Z(R, a(0))=W(a(0))+E(a(0))+\left\langle W^{(1)}(a(0))+E^{(1)}(a(0)),(R-a(0))\right\rangle \\
+\frac{1}{2}\left\langle(R-a(0)),\left[W^{(2)}(a(0))+E^{(2)}(a(0))\right](R-a(0))\right\rangle .
\end{gathered}
$$

By a simple estimate from [8],this substitution causes us to make another error of order $t \beta^{3 \alpha-1} / N$. Now we can explicitly compute

$$
\begin{aligned}
e^{-i t X(\beta) / \beta N} e^{-i t Z(R, a(0)) / \beta N} & \\
& \cdot \psi_{\alpha}(A(0), B(0), \beta, a(0), \eta(0), R) \phi_{1}(R+t \eta(0) / N, \beta \eta(0), r) \\
= & e^{-i t X(\beta) / \beta N} e^{-i t[W(a(0))+E(a(0))] / \beta N} \\
& \cdot \psi_{\alpha}\left(A(0), B_{N}(1), \beta, a(0), \eta_{N}(1), R\right) \phi_{1}(R+t \eta(0) / N, \beta \eta(0), r),
\end{aligned}
$$

where

$$
\eta_{N}(1)=\eta(0)-\left[W^{(1)}(a(0))+E^{(1)}(a(0))\right] t / N
$$

and

$$
B_{N}(1)=B(0)+2 i \beta^{2 \alpha-1}\left[W^{(2)}(a(0))+E^{(2)}(a(0))\right] A(0) t / N .
$$

Finally, a careful analysis of the action of $e^{-i t X(\beta) / \beta N}$ shows that we can approximate the right hand side of (3.5) up to an error of order $t \beta^{3 \alpha-1} / N$ by

$$
e^{i S_{N}(1) / \beta} \psi_{\alpha}\left(A_{N}(1), B_{N}(1), \beta, a_{N}(1), \eta_{N}(1), R\right) \phi_{1}\left(R, \beta \eta_{N}(1), r\right),
$$

where

$$
\begin{aligned}
& a_{N}(1)=a(0)+\eta_{N}(1) t / N, \\
& A_{N}(1)=A(0)+i \beta^{1-2 \alpha} B_{N}(1) t / 2 N,
\end{aligned}
$$$$
S_{N}(1)=\left[\eta_{N}(1)\right]^{2} t / 2 N-W(a(0)) t / N-E(a(0)) t / N .
$$

We now repeat this approximation procedure for each of the remaining 
$N-1$ factors from the Trotter product. This shows that we may approximate

$$
\begin{aligned}
& \left\{e^{-i t X(\beta) / \beta N} e^{-i t W / \beta N} e^{-i t h / \beta N}\right\}^{\ell} \\
& \quad \cdot \psi_{\alpha}(A(0), B(0), \beta, a(0), \eta(0), R) \phi_{1}(R, \beta \eta(0), r)
\end{aligned}
$$

by

$$
e^{i S_{N}(\ell) / \beta} \psi_{\alpha}\left(A_{N}(\ell), B_{N}(\ell), \beta, a_{N}(\ell), \eta_{N}(\ell), R\right) \phi_{1}\left(R, \beta \eta_{N}(\ell), r\right),
$$

where

$$
\begin{aligned}
& \eta_{N}(\ell)=\eta(0)-\sum_{j=1}^{\ell}\left[W^{(1)}\left(a_{N}(j-1)\right)+E^{(1)}\left(a_{N}(j-1)\right)\right] t / N \\
& a_{N}(\ell)=a(0)+\sum_{j=1}^{\ell} \eta_{N}(j) t / N \\
& A_{N}(\ell)=A(0)+\sum_{j=1}^{\ell} i \beta^{1-2 \alpha} B_{N}(j) t / 2 N \\
& B_{N}(\ell)=B(0)+\sum_{j=1}^{\ell} 2 i \beta^{2 \alpha-1}\left[W^{(2)}\left(a_{N}(j-1)\right)+E^{(2)}\left(a_{N}(j-1)\right)\right] A_{N}(j-1) t / N, \\
& S_{N}(\ell)=\sum_{j=1}^{\ell}\left\{\left[\eta_{N}(j)\right]^{2} / 2-W\left(a_{N}(j-1)\right)-E\left(a_{N}(j-1)\right)\right\} t / N
\end{aligned}
$$

Each step of the iteration costs us an error which is on the order of $t \beta^{3 \alpha-1} / N$.

After the $N$ th iteration we use (3.2) and (3.3) to replace

$$
e^{i S_{N}(N) / \beta} \psi_{\alpha}\left(A_{N}(N), B_{N}(N), \beta, a_{N}(N), \eta_{N}(N), R\right) \phi_{1}\left(R, \beta \eta_{N}(N), r\right)
$$

by

$$
e^{i S_{N}(N) / \beta} \psi_{\alpha}\left(A_{N}(N), B_{N}(N), \beta, a_{N}(N), \eta_{N}(N), R\right) \phi(R, r) .
$$

The errors we have made are on the order of $\beta^{3 \alpha-1}+N\left[t \beta^{3 \alpha-1} / N\right]+\beta^{3 \alpha-1} \leqq$ $C \beta^{3 \alpha-1}$ for $t \in[0, T]$ and arbitrarily large $N$. As we take $N$ to infinity, $a_{N}(N)$, $\eta_{N}(N), S_{N}(N), A_{N}(N)$, and $B_{N}(N)$ approach the solutions to (2.5)-(2.9). From this we obtain the theorem.

Remark. The strategy of the proof is to get an understanding of the short time behavior, and then to use an iteration to understand long times. We can do this because each iteration has an error on the order of $t \beta^{3 \alpha-1} / N$. If one tries to mimic our proof with $\phi$ in place of $\phi_{1}$, the short time errors will be on the order of $\operatorname{Min}\left\{t / N, \beta^{3 \alpha-1}\right\}$, but not $t \beta^{3 \alpha-1} / N$ (see the example in Part B below). For this reason we make use of $\phi_{1}$ in the proof.

\section{B. The Choice of $\phi_{1}$}

There are two approaches which one may use for determining a suitable vector $\phi_{1}(R, \beta \eta, r)$.

In the first method (which we shall not use), one makes the ansatz that the 
required $\phi_{1}$ can be written as

$$
\phi_{1}(\beta, R, \eta, r)=\phi(R, r)+\beta \tilde{\Phi}_{[1]}(R, \eta, r)+\beta^{2} \tilde{\Phi}_{[2]}(R, \eta, r)+\ldots
$$

One then substitutes this expression into the equation

$$
e^{-i t h(R) / \beta} \phi_{1}(\beta, R, \eta, r)=e^{-i t E(R) / \beta} \phi_{1}(\beta, R+s \eta, \eta, r)+0(t)
$$

for small $t$, and keeps terms up to order $\beta$. By taking time derivatives at $t=0$, one finds that $\widetilde{\Phi}_{[1]}$ must satisfy $(h(R)-E(R)) \widetilde{\Phi}_{[1]}(R, \eta, r)=\eta \cdot \nabla_{R} \phi(R, r)$. This equation for $\widetilde{\Phi}_{[1]}$ is easily solved by inverting the restriction of $h(R)-E(R)$ to the Hilbert space Ran $(1-P(R))$.

The second approach (which we will adopt) is motivated by Kato's proof of the adiabatic theorem $[12,15]$. If all of the vectors $\phi(\beta, R, \cdot)$ were independent of $R$, then we would have $e^{-i t h(R) / \beta} \phi(R, r)=e^{-i t E(R) / \beta} \phi\left(R^{\prime}, r\right)$ for any $R^{\prime}$, and (3.1) would trivially be satisfied with $\phi_{1}=\phi$. This situation is very similar to the one dealt with by Kato's adiabatic theorem $[12,15]$. The only difference is that in Kato's theorem, the Hamiltonians and eigenvectors depend on time rather than some other variable.

By mimicking a construction of Kato $[12 ; 13$, Sect. II-4; 18, p. 23], we begin our search for $\phi_{1}$ by choosing a unitary operator $U(R, \eta, s)$ such that $U(R, \eta, s) \phi(R, r)=\phi(R+s \eta, r)$. This operator satisfies the differential equation $i \frac{d}{d s} U(R, \eta, s)=A(R+s \eta, \eta) U(R, \eta, s)$, where $A(R, \eta)$ is the bounded self-adjoint operator $A(R, \eta)=i\left[\eta \cdot P^{(1)}(R), P(R)\right]([$,$] denotes the commutator).$

Standard techniques show that $U(R, \eta, s)$ is well approximated by $e^{-i s A(R, \eta)}$ for small $s$ if $R$ and $\eta$ lie in fixed compact sets. So, up to a small error we can approximate $\phi(R+s \eta, r)$ by $e^{-i s A(R, \eta)} \phi(R, r)$ for small $s$. Furthermore, $e^{-i s h(R) / \beta}$ is approximately equal to $e^{-i s A(R, \eta)} e^{-i s K(R, \beta \eta) / \beta}$ for small $s$ if we define

$$
K(R, \beta \eta)=h(R)-\beta A(R, \eta) .
$$

By ordinary perturbation theory, $K(R, \beta \eta)$ has a unique isolated eigenvalue $E_{1}(R, \beta \eta)$ near $E(R)$ for $\eta$ in a compact set and all small $\beta$. We choose $\phi_{1}(R, \beta \eta, r)$ to be the corresponding eigenfunction. It is determined up to a phase factor which we fix in Sect. 4.

Conditions (3.2) and (3.3) are easy to verify for this $\phi_{1}(R, \beta \eta, r)$. Condition (3.1) is harder to check. Since $\phi(R+s \eta, r)$ approximately equals $e^{-i s A(R, \eta)} \phi(R, r)$ and $\phi_{1}(R, \beta \eta, r)$ is near to $\phi(R, r)$ in $L^{2}\left(\mathbb{R}^{(n-k) m}, d r\right)$ for small $\beta$, we will be able to prove $\left\|\phi_{1}(R+s \eta, \beta \eta, \cdot)-e^{-i s A(R, \eta)} \phi_{1}(R, \beta \eta, \cdot)\right\|$ is bounded by a constant multiple of $\beta|s|$ for small $s$ and $\beta$. We will also show that $\left|E_{1}(R, \beta \eta)-E(R)\right|$ is dominated by a constant times $\beta^{2}$ for $R$ and $\eta$ in compact sets. From these facts we will see that $e^{-i s h(R) / \beta} \phi_{1}(R, \beta \eta, r)$ approximately equals $e^{-i S A(R, \eta)} e^{-i s K(R, \beta \eta) / \beta} \phi_{1}(R, \beta \eta, r)=e^{-i s E_{1}(R, \beta \eta) / \beta} e^{-i s A(R, \eta)} \phi_{1}(R, \beta \eta, r)$, which approximately equals $e^{-i s E(R) / \beta} \phi_{1}(R+s \eta, \beta \eta, r)$. This argument will establish (3.1).

We conclude this section with an instructive example of Kato's adiabatic theorem which illustrates some of the above ideas. The Hilbert space for this example is $\mathbb{C}^{2}$, and we study solutions to the equation $i \beta \frac{d}{d t} \psi(\beta, t)=H(t) \psi(\beta, t)$ for 
small $\beta$. We take $H(t)$ to be the matrix

$$
H(t)=\left[\begin{array}{cc}
\cos ^{2} t & \cos t \sin t \\
\cos t \sin t & \sin ^{2} t
\end{array}\right],
$$

which is the orthogonal projection onto the state

$$
\left[\begin{array}{c}
\cos t \\
\sin t
\end{array}\right] \text {. }
$$

Kato's theorem $[12,15]$ shows that if

$$
\psi(\beta, 0)=\left[\begin{array}{l}
1 \\
0
\end{array}\right]
$$

then

$$
\psi(\beta, t)=\left[\begin{array}{l}
e^{-i t / \beta} \cos t \\
e^{-i t / \beta} \sin t
\end{array}\right]+0(\beta) .
$$

As with our theorem, this is a zeroth order approximation.

As we mentioned earlier, there are two methods of obtaining a first order approximation. The first method involves assuming there is a solution of the form

$$
\widetilde{\Psi}_{1}(\beta, t)=e^{-i t / \beta}\left(\left[\begin{array}{c}
\cos t \\
\sin t
\end{array}\right]+\beta \psi_{[1]}(t)+\beta^{2} \psi_{[2]}(t)+\ldots\right) .
$$

One then derives equations which must be satisfied by the $\psi_{[j]}$ and keeps terms up to the desired order. The second method is the method we use for finding $\phi_{1}(R, \beta \eta, r)$, and for our very simple two dimensional example, it yields the exact solution.

The Hamiltonian $H(t)$ is equal to $e^{-i t A} H(0) e^{i t A}$, where $A$ is the commutator $A=i\left[\frac{d}{d t} H(t), H(t)\right]=\left[\begin{array}{rr}0 & -i \\ i & 0\end{array}\right]$, and $e^{-i t A}=\left[\begin{array}{rr}\cos t & -\sin t \\ \sin t & \cos t\end{array}\right]$. This $A$ is of course the analog of $A(R, \eta)$ for the molecular problem. The analog of $K(R, \beta \eta)$ is $K(\beta)=H(0)-\beta A=\left[\begin{array}{cc}1 & i \beta \\ -i \beta & 0\end{array}\right]$; the analog of $E_{1}(R, \beta \eta)$ is the eigenvalue $\frac{1}{2}+\frac{1}{2}\left(1+4 \beta^{2}\right)^{1 / 2}=1+\beta^{2}-\beta^{4}+\ldots$; and the analog of $\phi_{1}(R, \beta \eta, r)$ is the corresponding eigenfunction $\psi_{1}(\beta)$ of $K(\beta)$.

The exact solution to our simple system is $\psi(\beta, t)=e^{-i t A} e^{-i t K(\beta) / \beta} \psi(\beta, 0)$. So, we can easily verify (3.11) as follows:

$$
\begin{aligned}
\psi(\beta, t) & =e^{-i t A} e^{-i t K(\beta) / \beta} \psi(\beta, 0) \\
& =e^{-i t A} e^{-i t K(\beta) / \beta} \psi_{1}(\beta)+0(\beta) \\
& =e^{-i t\left[1+\beta^{2}-\beta^{4}+\ldots\right] / \beta} e^{-i t A} \psi_{1}(\beta)+0(\beta) \\
& =e^{-i t / \beta} e^{-i t A} \psi_{1}(\beta)+0(\beta) \\
& =e^{-i t / \beta} e^{-i t A} \psi(\beta, 0)+0(\beta) \\
& =\left[\begin{array}{c}
e^{-i t / \beta} \cos t \\
e^{-i t / \beta} \sin t
\end{array}\right]+0(\beta) .
\end{aligned}
$$


One may also check that for small times $t$, the error in $(3.11)$ is not $0(t \beta)$ by explicit calculation.

\section{Proof of Theorem 2.1}

In this section we present the details of the argument which was discussed in Sect. 3. Lemmas 4.1-4.7 establish technical results with which we prove Proposition 4.8. This proposition contains the crucial estimate which we use to prove Theorem 2.1.

The lemmas contain many constants, and we warn the reader that constants appearing in one lemma may implicitly depend on constants which occur in earlier lemmas.

Definition. For $0 \leqq t \leqq T$ we have $a(t) \in U$. Since $U$ is open and $a(t)$ is continuous, we can choose open $\bar{U}_{1}$ and $U_{2}$ such that their closures $\bar{U}_{1}$ and $\bar{U}_{2}$ are compact, $\bar{U}_{1} \subseteq U_{2}, \bar{U}_{2} \subseteq U$, and $a(t) \in U_{1}$ for $0 \leqq t \leqq T$. We choose $U_{1}$ and $U_{2}$ permanently.

Definition. Since $\bar{U}_{1}$ is a compact subset of the open set $U_{2}$, there exists $d>0$ such that $a \in \bar{U}_{1}$ and $|R-a| \leqq 2 d$ imply $R \in U_{2}$. We choose a function $F:[0, \infty) \rightarrow$ $[0,1]$ such that

$$
F(y)= \begin{cases}1 & \text { if } 0 \leqq y \leqq d \\ 0 & \text { if } 2 d \leqq y\end{cases}
$$

Definition. Suppose $R \in U$ and $\eta \in \mathbb{R}^{k m}$. Since $P(R)$ is analytic for $R \in U$, the operator $\eta \cdot P^{(1)}(R)$ is bounded and depends smoothly on $\eta$ and $R$. We define $A(R, \eta)=$ $i\left[\eta \cdot P^{(1)}(R), P(R)\right]$, where $[$,$] denotes the commutator. A(R, \eta)$ is bounded and self-adjoint. For each $R \in U$, and $\xi \in \mathbb{R}^{k m}$ we define $K(R, \xi)=h(R)-A(R, \xi)$ on $L^{2}\left(\mathbb{R}^{(n-k) m}\right) . K(R, \xi)$ is self-adjoint on the domain of $h(R)$.

Given $C_{0}$, there exists $b>0$ such that $b \leqq d^{3}(d$ is chosen in the definition of $F)$, and $0 \leqq \beta \leqq b,|\xi| \leqq b C_{0}, R \in \bar{U}_{2}$ imply that $K(R, \xi)$ has an isolated nondegenerate eigenvalue $E_{1}(R, \xi)$ such that $\lim _{\xi \rightarrow 0} E_{1}(R, \xi)=E(R)$. Both $E_{1}(R, \xi)$ and the associated projection $P_{1}(R, \xi)$ are analytic functions of each of their variables.

We now come to our first lemma which is a localized version of Lemma 2.3 of $[8]$.

Lemma 4.1. Suppose $\frac{1}{3}<\alpha \leqq \frac{1}{2}$. Define $\lambda=3 \alpha-1$ and set

$$
Y(R)=\left\{\begin{array}{cc}
W(R)+E(R) & \text { if } R \in \bar{U}_{2} \\
0 & \text { otherwise. }
\end{array}\right.
$$

For $R \in \mathbb{R}^{k m}$, and $a \in \bar{U}_{2}$ define

$$
Z(R, a)=Y(a)+\left\langle Y^{(1)}(a),(R-a)\right\rangle+\frac{1}{2}\left\langle(R-a), Y^{(2)}(a)(R-a)\right\rangle .
$$

For each $\Gamma>0$ there exists $C_{1}$ such that $\beta \in[0, b], a \in \bar{U}_{1}$, and $\|A\| \leqq \Gamma$ imply

$$
\left\|\left\{e^{-i s Y(R) / \beta}-e^{-i s Z(R, a) / \beta}\right\} F(|R-a|) \psi_{\alpha}(A, B, \beta, a, \eta, R)\right\| \leqq C_{1}|s| \beta^{\lambda}
$$

and

$$
\left\|\psi_{\alpha}(A, B, \beta, a, \eta, R)-F(|R-a|) \psi_{\alpha}(A, B, \beta, a, \eta, R)\right\| \leqq C_{1} \beta^{\lambda} .
$$


Proof. The second result is trivial, so we only prove the first.

Since $Y^{(2)}(R)$ is uniformly Lipshitz in $R$ for $R \in \bar{U}_{2}$, there exists $c$ such that $a \in \bar{U}_{1}$ and $|R-a| \leqq 2 d$ imply

$$
|Y(R)-Z(R, a)| \leqq c|R-a|^{3} \text {. }
$$

So, for $\beta \in[0, b]$ and $a \in \bar{U}_{1}$, we have

$$
\begin{aligned}
\| & \left\{e^{-i s Y(R) / \beta}-e^{i s Z(R, a) / \beta}\right\} F(|R-a|) \psi_{\alpha}(A, B, \beta, a, \eta, R) \|_{L^{2}(d R)} \\
& \leqq \beta^{-1}|s|\left\||Y(R)-Z(R, a)| F(|R-a|) \psi_{\alpha}(A, B, \beta, a, \eta, R)\right\|_{L^{2}(d R)} \\
& \leqq \beta^{-1} c|s|\left\|F(|R-a|)|R-a|^{3} \psi_{\alpha}(A, B, \beta, a, \eta, R)\right\|_{L^{2}(d R)} \\
& \leqq \beta^{-1} c|s|\left\||R-a|^{3} \psi_{\alpha}(A, B, \beta, a, \eta, R)\right\|_{L^{2}(d R)} \\
& =C_{1} \beta^{3 \alpha-1}|s|
\end{aligned}
$$

by explicit computation.

Lemma 4.2. Given $\varepsilon>0$ and $C_{0}$, there exists $\sigma_{1}>0$ such that

$$
\left\|\phi(R+s \eta, \cdot)-e^{-i s A(R, \eta)} \phi(R, \cdot)\right\| \leqq \varepsilon|s| / 9 T
$$

whenever $|s|<\sigma_{1}, R \in \bar{U}_{1},|\eta| \leqq C_{0}$

Proof. There exists $\gamma>0$ such that $|s| \leqq \gamma, R \in \bar{U}_{1}$, and $|\eta| \leqq C_{0}$ imply $R+s \eta \in \bar{U}_{2}$. For $|s| \leqq \gamma, R \in \bar{U}_{1}$, and $|\eta| \leqq C_{0}$ there exists [12;13, Sect. II-4; 18, p. 23] a unique unitary operator valued solution $U(R, \eta, s)$ to the equation $i \frac{d}{d s} U(R, \eta, s)=$ $A(R+s \eta, \eta) U(R, \eta, s)$ which satisfies the initial condition $U(R, \eta, s)=I$. The importance of this solution stems from the relation $P(R+s \eta) U(R, \eta, s)=$ $U(R, \eta, s) P(R)$, which is proved in $[12 ; 13$, Sect. II-4; 18, p. 23].

This relation implies $U(R, \eta, s) \phi(R, r)=e^{i \theta(R, \eta, s)} \phi(R+s \eta, r)$ for some real differentiable function $\theta(R, \eta, s)$ which satisfies $\theta(R, \eta, 0)=0$. Since $-i A(R, \eta, s)$ is a real bounded operator, $U(R, \eta, s)$ is also a real operator. So, $\theta(R, \eta, s)=0$ for all $s$.

If we define $Q(R, \eta, 0)=0$ and $Q(R, \eta, s)=s^{-1}\left[U(R, \eta, s)-e^{-i s A(R, \eta)}\right]$ for $s \neq 0$, then $Q(R, \eta, s)$ is continuous in $R, \eta$, and $s$. It is therefore uniformly continuous when restricted to the compact set $\left\{(R, \eta, s): R \in \bar{U}_{1},|\eta| \in C_{0}\right.$, and $\left.|s| \leqq \gamma\right\}$. This implies the lemma.

Using the construction of $[13$, Sect. II-4, Remark 4.4] we can construct an analytic operator valued function $U_{1}(R, \xi)$ such that $U_{1}(R, \xi)$ is unitary for $|\xi| \leqq b C_{0}$, and $R \in \bar{U}_{2} ; U_{1}\left(R_{0}, 0\right)=I$ for some $R_{0} \in U_{2} ;$ and $P_{1}(R, \xi) U_{1}(R, \xi)=$ $U_{1}(R, \xi) P_{1}\left(R_{0}, 0\right)$. We define $\phi_{1}(R, \eta, r)$ by $\phi_{1}(R, \eta, \cdot)=U_{1}(R, \cdot) \phi\left(R_{0}, 0, \cdot\right)$. One can easily prove $\phi_{1}(R, 0, r)=\phi(R, r)$ and that $\phi_{1}(R, \xi, \cdot)$ is normalized for $R \in \bar{U}_{2}$, and $|\xi| \leqq b C_{0}$.

Lemma 4.3. Given $C_{0}>0$, there exist $C_{2}$ and $C_{3}$ such that $\beta \in[0, b], R \in \bar{U}_{1}$, and $|\eta| \leqq C_{0}$ imply

$$
\left\|\phi_{1}(R, \beta \eta, \cdot)-\phi(R, \cdot)\right\| \leqq C_{2} \beta
$$


and

$$
\left|E_{1}(R, \beta \eta)-E(R)\right| \leqq C_{3} B^{2} .
$$

Proof. This follows from regular perturbation theory and a compactness argument. Since $P(R)$ is a projection, $P(R+s \eta)\left\{\frac{d}{d s} P(R+s \eta)\right\} P(R+s \eta)=0$. Thus

$$
\langle\phi(R+s \eta), A(R+s \eta \cdot \eta) \phi(R+s \eta)\rangle=0,
$$

and the first order contribution to $E_{1}(R, \beta \eta)-E(R)$ vanishes.

Lemma 4.4. Given $C_{0}>0$, there exists $C_{4}$ such that $\beta \in[0, b], R \in \bar{U}_{1},|\eta| \leqq C_{0}$, and $\left|\eta^{\prime}\right| \leqq C_{0}$ imply

$$
\left\|\phi_{1}(R, \beta \eta)-\phi_{1}\left(R, \beta \eta^{\prime}\right)\right\| \leqq C_{4} \beta\left|\eta-\eta^{\prime}\right| .
$$

Proof. This lemma follows from the uniform boundedness of $\nabla_{\xi} \phi_{1}(R, \xi)$ on the compact set $\left\{(R, \xi): R \in \bar{U}_{1},|\xi| \leqq b C_{0}\right\}$.

Lemma 4.5. Given $\varepsilon>0$ and $C_{0}$, there exist $C_{5}, C_{6}$, and $\sigma_{2} \in\left(0, \sigma_{1}\right]$ such that $\beta \in[0, b],|\eta| \leqq C_{0}, R \in \bar{U}_{1}$, and $|s| \leqq \sigma_{2}$ imply

$$
\left\|\phi_{1}(R+s \eta, \beta \eta)-e^{-i s A(R, \eta)} \phi_{1}(R, \beta \eta)\right\| \leqq\left\{C_{5} \beta+\varepsilon / 9 T\right\}|s|+C_{6} \beta|s|^{2} .
$$

Proof. Due to our choices of $U_{1}$ and $U_{2}$, there exists $\sigma_{2} \in\left(0, \sigma_{1}\right]$ such that $R \in \bar{U}_{1}$, $|s| \leqq \sigma_{2}$, and $|\eta| \leqq C_{0}$ imply $R+s \eta \in U_{2}$. So, by Lemma $4.2,|s| \leqq \sigma_{2}, R \in \bar{U}_{1}$, $|\eta| \leqq C_{0}$, and $\beta \in[0, b]$ imply

$$
\begin{aligned}
& \left\|\phi_{1}(R+s \eta, \beta \eta)-e^{-i s A(R, \eta)} \phi_{1}(R, \beta \eta)\right\| \\
& \leqq \varepsilon|s| / 9 T+\|\left\{\phi_{1}(R+s \eta, \beta \eta)-\phi_{1}(R+s \eta, 0)\right\} \\
& -e^{-i s A(R, \eta)}\left\{\phi_{1}(R, \beta \eta)-\phi_{1}(R, 0)\right\} \| \text {. }
\end{aligned}
$$

However, due to the boundedness of $A(R, \eta)$ for $R \in \bar{U}_{1}$, and $|\eta| \leqq C_{0}$, there exists $C_{6}^{\prime}$ such that

$$
\left\|e^{-i s A(R, \eta)}-1+i s A(R, \eta)\right\| \leqq C_{6}^{\prime}|s|^{2} .
$$

Thus, the right hand side of (4.2) is bounded by

$$
\begin{aligned}
& \left\|\phi_{1}(R+s \eta, \beta \eta)-\phi_{1}(R+s \eta, 0)-\phi_{1}(R, \beta \eta)+\phi_{1}(R, 0)\right\| \\
& \quad+|s|\|A(R, \eta)\|\left\|\phi_{1}(R, \beta \eta)-\phi_{1}(R, 0)\right\| \\
& \quad+C_{6}^{\prime}|s|^{2}\left\|\phi_{1}(R, \beta \eta)-\phi_{1}(R, 0)\right\| \\
& \quad+\varepsilon|s| / 9 T .
\end{aligned}
$$

By Lemma 4.3 and the uniform boundedness of $A(R, \eta)$, this is bounded by

$$
\begin{aligned}
& \left\|\phi_{1}(R+s \eta, \beta \eta)-\phi_{1}(R+s \eta, 0)-\phi_{1}(R, \beta \eta)+\phi_{1}(R, 0)\right\| \\
& \quad+C_{5}^{\prime} \beta|s|+\varepsilon|s| / 9 T+C_{6}^{\prime} C_{2} \beta|s|^{2}
\end{aligned}
$$

for some $C_{5}^{\prime}$.

From this it is clearly sufficient for us to prove

$$
\left\|\phi_{1}(R+s \eta, \beta \eta)-\phi_{1}(R+s \eta, 0)-\phi_{1}(R, \beta \eta)+\phi_{1}(R, 0)\right\| \leqq C_{5}^{\prime \prime} \beta|s|
$$


for some $C_{5}^{\prime \prime}$. However, by compactness there exists $C_{5}^{\prime \prime \prime}$ such that $R+s \eta \in \bar{U}_{2}$ and $|\xi| \leqq b C_{0}$ imply $\left\|\frac{\partial}{\partial s} \nabla_{\xi} \phi_{1}(R+s \eta, \xi)\right\| \leqq C_{5}^{\prime \prime \prime}$. So, by the fundamental theorem of calculus,

$$
\begin{aligned}
& \left\|\phi_{1}(R+s \eta, \beta \eta)-\phi_{1}(R+s \eta, 0)-\phi_{1}(R, \beta \eta)+\phi_{1}(R, 0)\right\| \\
& =\left\|\int_{0}^{s} \int_{0}^{\beta \eta}\left\{\frac{\partial}{\partial t} \nabla_{\xi} \phi_{1}(R+t \eta, \xi)\right\} \cdot d \xi d t\right\| \\
& \leqq C_{5}^{\prime \prime \prime} \beta|\eta||s| \\
& \leqq C_{5}^{\prime \prime \prime} C_{0} \beta|s| \\
& \leqq C_{5}^{\prime \prime} \beta|s| .
\end{aligned}
$$

Lemma 4.6. For $\beta \in[0, b], a \in \bar{U}_{1}$, and $|\eta| \leqq C_{0}$ we define $f(\beta, a, \eta, R, r)=$ $F(|R-a|) \phi_{1}(R, \beta \eta, r)$. Let $\Sigma_{1}$ be a compact set of pairs of matrices $(A, B)$ which satisfy conditions (2.1) - (2.4). Given $\varepsilon>0$ there exist $\sigma_{3} \in\left(0, \sigma_{2}\right], C_{7}$, and $C_{8}$ such that $0 \leqq \alpha \leqq \frac{1}{2},(A, B) \in \Sigma_{1}, \beta \in(0, b], a \in \bar{U}_{1},|\eta| \leqq C_{0}$, and $|s| \leqq \sigma_{3}$ imply

$$
\begin{aligned}
& \| e^{-i s X(\beta) / \beta} f(\beta, a, \eta, R, r) \psi_{\alpha}(A, B, \beta, a, 0, R) \\
& \quad-f(\beta, a, \eta, R, r) e^{-i s X(\beta) / \beta} \psi_{\alpha}(A, B, \beta, a, 0, R) \|_{L^{2}(d R d r)} \\
& \quad \leqq C_{7} \beta|s|+C_{8} \beta^{1-\alpha}|s|+\varepsilon \beta^{1-2 \alpha}|s| /\left(9 T b^{1-2 \alpha}\right) .
\end{aligned}
$$

Proof. Since $a \in \bar{U}_{1}$ plays no essential role, we assume for convenience that $0 \in \bar{U}_{1}$ and $a=0$. Next we apply a unitary dilation to replace $R$ by the variable $q=\beta^{-\alpha} R$. In this representation the conclusion we must prove can be stated as follows: For some $\sigma_{3} \in\left(0, \sigma_{2}\right], C_{7}$, and $C_{8}$, the following inequality holds for $0 \leqq \alpha \leqq \frac{1}{2}$, $(A, B) \in \Sigma_{1}, \beta \in[0, b], a \in \bar{U}_{1}$, and $|s| \leqq \sigma_{3}$ :

$$
\begin{aligned}
& \| e^{i s \beta^{1-2 \alpha} \Delta_{q} / 2} f\left(\beta, a, \eta, \beta^{\alpha} q, r\right) \psi_{\alpha}(A, B, 1,0,0, q) \\
& \quad-f\left(\beta, a, \eta, \beta^{\alpha} q, r\right) e^{i s \beta^{1}{ }^{2 \alpha} \Delta_{q} / 2} \psi_{\alpha}(A, B, 1,0,0, q) \|_{L^{2}(d r d q)} \\
& \quad \leqq C_{7} \beta|s|+C_{8} \beta^{1-\alpha}|s|+\varepsilon \beta^{1-2 \alpha}|s| /\left(9 T b^{1-2 \alpha}\right) .
\end{aligned}
$$

To prove this we first note that $\left\{f\left(\beta, a, \eta, \beta^{\alpha} q, r\right) \psi_{\alpha}(A, B, 1,0,0, R):(A, B) \in \Sigma_{1}\right.$, $\beta \in[0, b], a \in \bar{U}_{1}$, and $\left.|\eta| \leqq C_{0}\right\}$ is a compact subset of the Banach space $\mathscr{A}=\left\{g(R, r):\|g\|_{L^{2}(d r d q)}+\left\|\Delta_{q} g\right\|_{L^{2}(d r d q)}<\infty\right\}$. Standard argument [16, pp. 295296] now show that there exists $\sigma_{3} \in\left(0, \sigma_{2}\right]$ such that $\beta \in[0, b],(A, B) \in \Sigma_{1}, a \in \bar{U}_{1}$, $|\eta| \leqq C_{0}$, and $|t| \leqq \sigma_{3} b^{1-2 \alpha}$ imply

$$
\begin{gathered}
\| e^{i t \Delta / 2} f\left(\beta, a, \eta, \beta^{\alpha} q, r\right) \psi_{\alpha}(A, B, 1,0,0, q)-f\left(\beta, a, \eta, \beta^{\alpha} q, r\right) \psi_{\alpha}(A, B, 1,0,0, q) \\
-\frac{1}{2} i t \Delta f\left(\beta, a, \eta, \beta^{\alpha} q, r\right) \psi_{\alpha}(A, B, 1,0,0, q) \|_{L^{2}(d r d q)} \leqq \varepsilon|t| /\left(18 T b^{1-2 \alpha}\right)
\end{gathered}
$$

and

$$
\begin{aligned}
& \left\|e^{i t \Delta / 2} \psi_{\alpha}(A, B, 1,0,0, q)-\psi_{\alpha}(A, B, 1,0,0, q)-\frac{1}{2} i t \Delta \psi_{\alpha}(A, B, 1,0,0, q)\right\|_{L^{2}(d q)} \\
& \quad \leqq \varepsilon|t| /\left(18 T b^{1-2 \alpha}\right) .
\end{aligned}
$$

The left hand side of (4.3) is bounded by

$$
\begin{aligned}
& \left\|\left\{e^{i s \beta^{1-2 \alpha} \Delta / 2}-1-i s \beta^{1-2 \alpha} \Delta / 2\right\} f\left(\beta, a, \eta, \beta^{\alpha} q, r\right) \psi_{\alpha}(A, B, 1,0,0, q)\right\|_{L^{\cdot}(d r d q)} \\
& +\left\|f\left(\beta, a, \eta, \beta^{\alpha} q, r\right)\left\{1+i s \beta^{1-2 \alpha} \Delta / 2-e^{i s \beta^{1-2 \alpha} \Delta / 2}\right\} \psi_{\alpha}(A, B, 1,0,0, q)\right\|_{L^{2}(d r d q)}
\end{aligned}
$$




$$
\begin{aligned}
& +|s| \beta^{1-2 \alpha}\left\|\left\{\nabla_{q} f\left(\beta, a, \eta, \beta^{\alpha} q, r\right)\right\} \cdot \nabla_{q} \psi_{\alpha}(A, B, 1,0,0, q)\right\|_{L^{2}(d r d q)} / 2 \\
& +|s| \beta^{1-2 \alpha}\left\|\left\{\Delta_{q} f\left(\beta, a, \eta, \beta^{\alpha} q, r\right)\right\} \psi_{\alpha}(A, B, 1,0,0, q)\right\|_{L^{2}(d r d q)} / 2 .
\end{aligned}
$$

For $(A, B) \in \Sigma_{1}, \beta \in[0, b], a \in \bar{U}_{1},|\eta| \leqq C_{0}$, and $|s| \leqq \sigma_{3}$, the sum of the first two terms of (4.4) is bounded by $\varepsilon \beta^{1-2 \alpha}|s| /\left(9 T b^{1-2 \alpha}\right)$. The third is bounded by $|s| \beta^{1-\alpha}\left\|\left\{\left\|\nabla_{R} f(\beta, a, \eta, R, r)\right\|_{L^{2}(d r)}\right\}\right\|_{L^{\infty}(d R)}\left\|\nabla_{q} \psi_{\alpha}(A, B, 1,0,0, q)\right\|_{L^{2}(d q)}$. The fourth is bounded by $\beta|s|\left\|\left\{\left\|\Delta_{R} f(\beta, a, \eta, R, r)\right\|_{L^{2}(d r)}\right\}\right\|_{L^{\infty}(d R)}$. These bounds imply the lemma.

\section{Remarks}

1. Lemma 4.6 is false if we replace $\psi_{\alpha}(A, B, \beta, a, 0, R)$ by $\psi_{\alpha}(A, B, \beta, a, \eta, R)$ with some $\eta \neq 0$. Under the unitary scaling used in the proof, $\psi_{\alpha}(A, B, \beta, 0, \eta, R)$ transforms into $\psi_{\alpha}\left(A, B, 1,0, \beta^{\alpha-1} \eta, q\right)$. The presence of the factor $e^{i \beta^{\alpha-1}\langle\eta, q\rangle}$ destroys our compactness argument and causes the bound on the third term of (4.4) to behave like a constant times $|s|$ rather than $C_{8}|s| \beta^{1-\alpha}$.

2. In spite of the above remark, we can still use Lemma 4.6 to estimate $e^{-i s X(\beta) / \beta} f(\beta, a, \eta, R, r) \psi_{\alpha}(A, B, \beta, a, \eta, R)$. The following computation illustrates the required ideas:

$$
\begin{aligned}
e^{-i s X(\beta) / \beta} f(\beta, a, \eta, R, r) \psi_{\alpha}(A, B, \beta, a, \eta, R) \\
\quad=e^{i s \beta \Delta / 2} e^{i\langle\eta, R>/ \beta} f(\beta, a, \eta, R, r) \psi_{\alpha}(A, B, \beta, a, 0, R) \\
\quad=e^{i\langle\eta, R>/ \beta} e^{-s \eta \nabla} e^{i s \eta^{2} / 2 \beta} e^{i s \beta \Delta / 2} f(\beta, a, \eta, R, r) \psi_{\alpha}(A, B, \beta, a, 0, R) \\
\quad=e^{i\langle\eta, R>/ \beta} e^{i s \eta^{2} / 2 \beta} e^{-s \eta \cdot \nabla}\left\{f(\beta, a, \eta, R, r) e^{-i s X(\beta) / \beta} \psi_{\alpha}(A, B, \beta, a, 0, R)+\text { errors }\right\} \\
\quad=f(\beta, a, \eta, R-s \eta, r) e^{-i s X(\beta) / \beta} \psi_{\alpha}(A, B, \beta, a, \eta, R)+\text { errors. }
\end{aligned}
$$

By Lemma 4.6 and the unitarity of $e^{-s \eta \cdot \nabla}$, the errors satisfy the bounds in the conclusion to Lemma 4.6.

Lemma 4.7. For each $\varepsilon>0, \beta \in(0, b]$, and $C_{0}>0$, there exists $\sigma_{4} \in\left(0, \sigma_{3}\right]$ such that $R \in \bar{U}_{1},|\eta| \leqq C_{0}$, and $|s| \leqq \sigma_{4}$ imply

$$
\begin{aligned}
& \left\|e^{-i s h(R) / \beta} \phi_{1}(R, \beta \eta)-e^{-i s A(R, \eta)} e^{-i s K(R, \beta \eta) / \beta} \phi_{1}(R, \beta \eta)\right\| \\
& \quad \leqq \varepsilon|s| / 9 T .
\end{aligned}
$$

Proof. The operators $h(R)$ and $K(R, \beta \eta)$ all have the same domain $\mathscr{D}$. Furthermore, for some constant $c$, all of the norms $\|f\|_{1}=c\|f\|+\|h(R) f\|$, and $\|f\|_{2}=c\|f\|+\|K(R, \beta \eta) f\|$ are all equivalent on $\mathscr{D}$ for $R \in \bar{U}_{1}$ and $|\eta| \leqq C_{0}$. In the topology determined by these norms, $\left\{\phi_{1}(R, \beta \eta): R \in \bar{U}_{2},|\eta| \leqq C_{0}\right\}$ is a compact subset of $\mathscr{D}$.

The derivative at $s=0$ of $\left\{e^{-i s h(R) / \beta}-e^{-i s A(R, \eta)} e^{-i s K(R, \beta \eta) / \beta}\right\} \phi_{1}(R, \beta \eta)$ is zero. So, for each choice of $R$ and $\eta$ we can find a $\sigma_{4} \in\left(0, \sigma_{3}\right]$ such that (4.6) holds for $|s| \leqq \sigma_{4}$. The uniformity in $R$ and $\eta$ follows from the compactness noted above, the continuity in $R$ and $\eta$ of $A(R, \eta)$, and the continuity of $h(R)$ and $K(R, \beta \eta)$ in $R$ and $\eta$ as operators from their common domain into $L^{2}\left(\mathbb{R}^{(n-k) m}\right)$.

Proposition 4.8. (Consolidation of Lemmas 4.1-4.7) Suppose $\frac{1}{3}<\alpha \leqq \frac{1}{2}$ and $\lambda=$ $3 \alpha-1$. Let $\Sigma$ be any compact set of pairs of matrices $(A, B)$ which satisfy conditions 12.1)-12.4). Given $C_{n}>C_{n}^{\prime}>0$, there exists $C^{\prime}$ such that for each $\varepsilon>0$ and each 
fixed $\beta \in(0, b]$ there exists $\sigma_{5}>0$ such that $(A, B) \in \Sigma, a \in \bar{U}_{1},|\eta| \leqq C_{0}^{\prime}$, and $|s| \leqq \sigma_{5}$ imply

$$
\begin{aligned}
& \| e^{-i s X(\beta) / \beta} e^{-i s W(R) / \beta} e^{-i s h(R) / \beta} \psi_{\alpha}(A, B, \beta, a, \eta, R) F(|R-a|) \phi_{1}(R, \beta \eta) \\
& \quad-e^{i S_{1}(s) / \beta} \psi_{\alpha}\left(A_{1}(s), B_{1}(s), \beta, a_{1}(s), \eta_{1}(s), R\right) F\left(\left|R-a_{1}(s)\right|\right) \phi_{1}\left(R, \beta \eta_{1}(s) \|\right. \\
& \leqq \\
& \quad C^{\prime} \beta^{\lambda}|s| / T+\varepsilon|s| / 3 T .
\end{aligned}
$$

$A_{1}(s), B_{1}(s), a_{1}(s), \eta_{1}(s)$, and $S_{1}(s)$ are given by the following formulas:

$$
\begin{aligned}
& \eta_{1}(s)=\eta-s\left[E^{(1)}(a)+W^{(1)}(a)\right] \\
& a_{1}(s)=a+s \eta_{1}(s) \\
& S_{1}(s)=\left\{\left[\eta_{1}(s)\right]^{2} / 2-E(a)-W(a)\right\} s \\
& B_{1}(s)=B+2 i s \beta^{2 \alpha-1}\left[E^{(2)}(a)+W^{(2)}(a)\right] A \\
& A_{1}(s)=A+i s \beta^{1-2 \alpha} B_{1}(s) / 2 .
\end{aligned}
$$

Proof. Let $C_{9}=\sup \left\{\left|E^{(1)}(a)+W^{(1)}(a)\right|: a \in \bar{U}_{2}\right\}$. If $|s| \leqq\left(C_{0}-C_{0}^{\prime}\right) / C_{9}$, then $\left|\eta_{1}(s)\right| \leqq C_{0}$ for any $a \in \bar{U}_{2}$. Since $\Sigma$ is compact, there exists $\Gamma>0$ such that $(A, B) \in \Sigma$, $\beta \in[0, b]$, and $|s| \leqq\left(C_{0}-C_{0}^{\prime}\right) / C_{9}$ imply $\left\|A_{1}(s)\right\|=\| A+i \beta^{1-2 \alpha} s B / 2-s^{2}\left\{E^{(2)}(a)+\right.$ $\left.W^{(2)}(a)\right\} A \| \leqq \Gamma$.

We now fix $\beta \in(0, b]$. Then there exists a compact set $\Sigma_{1}$ of pairs of matrices which satisfy conditions $(2.1)-(2.4)$, such that $\left(A_{1}(s), B_{1}(s)\right) \in \Sigma_{1}$ for all choices of $(A, B) \in \Sigma, a \in \bar{U}_{1},|\eta| \leqq C_{0}^{\prime}$, and $|s| \leqq\left(C_{0}-C_{0}^{\prime}\right) / C_{9}$.

These choices of $\alpha, \lambda, C_{0}, \varepsilon$, and $\Sigma_{1}$ now determine the constants $\sigma_{4} \leqq \sigma_{3} \leqq \sigma_{2} \leqq$ $\sigma_{1} \leqq\left(C_{0}-C_{0}^{\prime}\right) / C_{9}$ and $C_{1}, C_{2}, \ldots, C_{8}$ which occur in Lemmas 4.1-4.7.

By Lemma $4.7, \mathrm{a} \in \bar{U}_{1},|\eta| \leqq C_{0}$, and $|s| \leqq \sigma_{4}$ imply

$$
\begin{aligned}
& \| e^{-i s h / \beta} \psi_{\alpha}(A, B, \beta, a, \eta, R) F(|R-a|) \phi_{1}(R, \beta \eta) \\
& \quad-\psi_{\alpha}(A, B, \beta, a, \eta, R) F(|R-a|) e^{-i s A(R, \eta)} e^{-i s K(R, \beta \eta) / \beta} \phi_{1}(R, \beta \eta) \| \\
& \quad \leqq \varepsilon|s| / 9 T .
\end{aligned}
$$

Under the same conditions on $a, \eta$, and $s$, Lemma 4.5 and the definition of $\phi_{1}(R, \beta \eta)$ show that

$$
\begin{aligned}
& \| \psi_{\alpha}(A, B, \beta, a, \eta, R) F(|R-a|) e^{-i s A(R, \eta)} e^{-i s K(R, \beta \eta) / \beta} \phi_{1}(R, \beta \eta) \\
& \quad-e^{-i s E_{1}(R, \beta \eta) / \beta} \psi_{\alpha}(A, B, \beta, a, \eta, R) F(|R-a|) \phi_{1}(R+s \eta, \beta \eta) \| \\
& \quad \leqq\left(C_{5} \beta+\varepsilon / 9 T\right)|s|+C_{6} \beta|s|^{2} .
\end{aligned}
$$

Next, using $\left|e^{-i s E_{1}(R, \beta \eta) / \beta}-e^{-i s E(R) / \beta}\right| \leqq|s|\left|E_{1}(R, \beta \eta)-E(R)\right| / \beta$ and Lemma 4.3, we have

$$
\begin{aligned}
& \| e^{-i s E_{1}(R, \beta \eta) / \beta} \psi_{\alpha}(A, B, \beta, a, \eta, R) F(|R-a|) \phi_{1}(R+s \eta, \beta \eta) \\
& \quad-e^{-i s E(R) / \beta} \psi_{\alpha}(A, B, \beta, a, \eta, R) F(|R-a|) \phi_{1}(R+s \eta, \beta \eta) \| \\
& \quad \leqq C_{3} \beta|s| .
\end{aligned}
$$

We now let $Z(R, a)$ denote the second order Taylor expansion of $W(R)+E(R)$ 
about the point $a$. By Lemma 4.1, $a \in \bar{U}_{1},(A, B) \in \Sigma$ and $|s| \leqq \sigma_{4}$ imply

$$
\begin{aligned}
& \| e^{-i s\{W(R)+E(R)\} / \beta} \psi_{\alpha}(A, B, \beta, a, \eta, R) F(|R-a|) \phi_{1}(R+s \eta, \beta \eta) \\
& \quad-e^{-i s Z(R, a) / \beta} \psi_{\alpha}(A, B, \beta, a, \eta, R) F(|R-a|) \phi_{1}(R+s \eta, \beta \eta) \| \\
& \quad \leqq C_{1}|s| \beta^{\lambda} .
\end{aligned}
$$

At this point we explicitly compute

$$
\begin{aligned}
& e^{-i s Z(R, a) / \beta} \psi_{\alpha}(A, B, \beta, a, \eta, R) F(|R-a|) \phi_{1}(R+s \eta, \beta \eta) \\
& \quad=e^{-i s[W(a)+E(a)] / \beta} \psi_{\alpha}\left(A, B_{1}(s), \beta, a, \eta_{1}(s), R\right) F(|R-a|) \phi_{1}(R+s \eta, \beta \eta) .
\end{aligned}
$$

Since $a \in \bar{U}_{1},\left|\eta_{1}(s)\right| \leqq C_{0},\left(A, B_{1}(s)\right) \in \Sigma_{1}$, and $|s| \leqq \sigma_{4}$, we can mimic the computation (4.5) and apply Lemma 4.6 to obtain

$$
\begin{aligned}
& \| e^{-i s X(\beta) / \beta} e^{-i s[W(a)+E(a)] / \beta} \psi_{\alpha}\left(A, B_{1}(s), \beta, a, \eta_{1}(s), R\right) F(|R-a|) \phi_{1}(R+s \eta, \beta \eta) \\
& \quad-e^{i S_{1}(s) / \beta} \psi_{\alpha}\left(A_{1}(s), B_{1}(s), \beta, a_{1}(s), \eta_{1}(s), R\right) \\
& \cdot F\left(\left|R-a_{1}(s)\right|\right) \phi_{1}\left(R+s \eta-s \eta_{1}(s), \beta \eta\right) \| \\
& \quad \leqq C_{7}|s| \beta+C_{8}|s| \beta^{1-\alpha}+\varepsilon|s| \beta^{1-2 \alpha} /\left(9 T b^{1-2 \alpha}\right) .
\end{aligned}
$$

Since $\phi_{1}(R, \beta \eta)$ is uniformly Lipshitz for $R \in \bar{U}_{2}$ and $\left|\eta-\eta_{1}(s)\right|$ is bounded by $C_{9}|s|$, there exists $C_{10}$ such that $\left\|\phi_{1}\left(R+s \eta-s \eta_{1}(s), \beta \eta\right)-\phi_{1}(R, \beta \eta)\right\| \leqq C_{10}|s|^{2}$. This and Lemma 4.4 now imply

$$
\begin{aligned}
& \| e^{i S_{1}(s) / \beta} \psi_{\alpha}\left(A_{1}(s), B_{1}(s), \beta, a_{1}(s), \eta_{1}(s), R\right) F\left(\left|R-a_{1}(s)\right|\right) \phi_{1}\left(R+s \eta-s \eta_{1}(s), \beta \eta\right) \\
& \quad-e^{i S_{1}(s) / \beta} \psi_{\alpha}\left(A_{1}(s), B_{1}(s), \beta, a_{1}(s), \eta_{1}(s), R\right) F\left(\left|R-a_{1}(s)\right|\right) \phi_{1}\left(R, \beta \eta_{1}(s)\right) \| \\
& \quad \leqq C_{10}|s|^{2}+C_{4} \beta C_{9}|s| .
\end{aligned}
$$

By using the unitarity of $e^{-i s X(\beta) / \beta}$ and $e^{-i s W / \beta}$, we can now combine (4.8)-(4.14) to obtain the following result: $(A, B) \in \Sigma, a \in \bar{U}_{1},|\eta| \leqq C_{0}^{\prime}$, and $|s| \leqq \sigma_{4}$ imply

$$
\begin{aligned}
& \| e^{-i s X(\beta) / \beta} e^{-i s W / \beta} e^{-i s h / \beta} \psi(A, B, \beta, a, \eta, R) F(|R-a|) \phi_{1}(R, \beta \eta) \\
& -e^{i S_{1}(s) / \beta} \psi_{\alpha}\left(A_{1}(s), B_{1}(s), \beta, a_{1}(s), \eta_{1}(s), R\right) F\left(\left|R-a_{1}(s)\right|\right) \phi_{1}\left(R, \beta \eta_{1}(s)\right) \| \\
& \leqq \\
& \quad\left\{\varepsilon / 9 T+C_{5} \beta+\varepsilon / 9 T+C_{6} \beta|s|+C_{3} \beta+C_{1} \beta^{\lambda}+C_{7} \beta+C_{8} \beta^{1-\alpha}+\varepsilon / 9 T\right. \\
& \left.\quad+C_{10}|s|+C_{4} C_{9} \beta\right\}|s| .
\end{aligned}
$$

This implies the lemma since we can choose $\sigma_{5} \leqq \operatorname{Min}\left\{\beta^{\lambda}, \sigma_{4}\right\}$

Proof of Theorem 2.1. We will use the Trotter Product Formula and Proposition 4.8 in tandem, and as a consequence we must consider the discrete time analogs (3.6)-(3.10) of the solution to equations (2.5)-(2.9). We choose $b>0$ as in the discussion at the beginning of this section and fix $t \in[0, T]$ and $\beta \in(0, b]$. Then by taking $N$ sufficiently large we can make

$$
\begin{aligned}
\mid a(\ell t / N) & -a_{N}(\ell)|+| \eta(\ell t / N)-\eta_{N}(\ell) \mid+\left\|A(\ell t / N)-A_{N}(\ell)\right\| \\
& +\left\|B(\ell t / N)-B_{N}(\ell)\right\|+\left|S(\ell t / N)-S_{N}(\ell)\right|
\end{aligned}
$$


arbitrarily small for $\ell=1,2, \ldots, N$ (see [1]). This has two consequences. First, we can choose $C_{0}>C_{0}^{\prime}, \Gamma, N_{1}$, and a compact set $\Sigma$ of pairs of matrices satisfying (2.1)-(2.4) such that the following conditions hold for all $N \geqq N_{1}$ and $\ell=0$, $1,2, \ldots, N: \quad a_{N}(\ell) \in \bar{U}_{1} ;|\eta(s)| \leqq C_{0}^{\prime}$ for $0 \leqq s \leqq t ;\left|\eta_{N}(\ell)\right| \leqq C_{0}^{\prime} ;\|A(s)\| \leqq \Gamma$ for $0 \leqq s \leqq t ;\left\|A_{N}(\ell)\right\| \leqq \Gamma ;(A(s), B(s)) \in \Sigma$ for $0 \leqq s \leqq t ;$ and $\left(A_{N}(\ell), B_{N}(\ell)\right) \in \Sigma$. The second consequence is that by the dominated convergence theorem, there exists $N_{2} \geqq N_{1}$ such that $N \geqq N_{2}$ implies

$$
\begin{aligned}
& \| e^{i S_{N}(N) / \beta} \psi_{\alpha}\left(A_{N}(N), B_{N}(N), \beta, a_{N}(N), \eta_{N}(N), \cdot\right) \\
& -e^{i S(t) / \beta} \psi_{\alpha}(A(t), B(t), \beta, a(t), \eta(t), \cdot) \| \leqq \varepsilon / 3,
\end{aligned}
$$

where $\varepsilon>0$ is arbitrary but fixed.

The Trotter Product Formula [16] shows the existence of $N_{3} \geqq N_{2}$ such that $N \geqq N_{3}$ implies

$$
\begin{aligned}
& \|\left[e^{-i t H(\beta) / \beta}-\left\{e^{-i t X(\beta) / \beta N} e^{-i t W / \beta N} e^{-i t h / \beta N}\right\}^{N}\right] \\
& \quad \cdot \psi_{\alpha}(A(0), B(0), \beta, a(0), \eta(0), R) F(|R-a(0)|) \phi_{1}(R, \beta \eta(0)) \|<\varepsilon / 3 .
\end{aligned}
$$

With $C_{0}, C_{0}^{\prime}, \Gamma, \Sigma$, and $\beta$ as above, we choose $\sigma_{5}$ so that the conclusion of Proposition 4.8 holds for $|s| \leqq \sigma_{5}$. Given $\sigma_{5}$, we next choose $N_{4} \geqq N_{3}$ so that $N \geqq N_{4}$ implies $T / N \leqq \sigma_{5}$. Then for $N \geqq N_{4}, 0 \leqq j \leqq N-1$, and $\ell=j+1$ we have

$$
\begin{aligned}
& \|\left\{e^{-i t X(\beta) / \beta N} e^{-i t W / \beta N} e^{-i t h / \beta N}\right\} e^{i S_{N}(j) / \beta} \psi_{\alpha}\left(A_{N}(j), B_{N}(j), \beta,\right. \\
& \left.a_{N}(j), \eta_{N}(j), R\right) F\left(\left|R-a_{N}(j)\right|\right) \phi_{1}\left(R, \beta \eta_{N}(j)\right) \\
& \quad-e^{i S_{N}(\ell) / \beta} \psi_{\alpha}\left(A_{N}(\ell), B_{N}(\ell), \beta\right. \\
& \left.a_{N}(\ell), \eta_{N}(\ell), R\right) F\left(\left|R-a_{N}(\ell)\right|\right) \phi_{1}\left(R, \beta \eta_{N}(\ell)\right) \| \\
& \quad \leqq C \beta^{\lambda} t / N+\varepsilon / 3 N .
\end{aligned}
$$

So, by using the triangle inequality, (4.15), (4.16), and (4.17) for all values of $j$, we have

$$
\begin{aligned}
& \| e^{-i t H(\beta) / \beta} \psi_{\alpha}(A(0), B(0), \beta, a(0), \eta(0), R) F(|R-a(0)|) \phi_{1}(R, \beta \eta(0)) \\
& \quad-e^{i S(t) / \beta} \psi_{\alpha}(A(t), B(t), \beta, a(t), \eta(t), R) F(|R-a(t)|) \phi_{1}(R, \beta \eta(t)) \| \\
& \leqq C \beta^{\lambda}+\varepsilon
\end{aligned}
$$

where $\varepsilon>0$ is arbitrary. By Lemma 4.3 we can replace both $\phi_{1}(R, \beta \eta(0))$ and $\phi_{1}(R, \beta \eta(t))$ in the last expression by $\phi(R)$ at the expense of increasing $C$. By increasing $C$ further, Lemma 4.1 shows that we may also replace both $F(|R-a(0)|)$ and $F(|R-a(t)|)$ by 1 . This implies the theorem. 


\section{References}

1. Birkhoff, G., Rota, G.-C. : Ordinary differential equations. London, Toronto, Waltham, Mass. : Blaisdell Publishing Company 1962

2. Born, M., Oppenheimer, R. : Zur Quantentheorie der Molekeln. Ann. Phys. (Leipzig) 84, 457-484 (1927)

3. Combes, J. M.: On the Born-Oppenheimer approximation. In: International symposium on mathematical problems in theoretical physics (ed. H. Araki), pp. 467-471. Berlin, Heidelberg, New York: Springer 1975

4. Combes, J. M. : The Born-Oppenheimer approximation. In: The Schrödinger equation (eds. W. Thirring, P. Urban), pp. 139-159. Wien, New York: Springer 1977

5. Combes, J. M., Seiler, R. : Regularity and asymptotic properties of the discrete spectrum of electronic Hamiltonians. Int. J. Quantum Chem. 14, 213-229 (1978)

6. Eyring, H.: The activated complex and the absolute rate of chemical reactions. Chem. Rev. 17, 65-77 (1935)

7. Hagedorn, G. A. : Semiclassical quantum mechanics for coherent states. In: The proceedings of the international conference on mathematical physics (ed. K. Osterwalder). Berlin, Heidelberg, New York: Springer (to appear 1980)

8. Hagedorn, G. A. : Semiclassical quantum mechanics I: The $h \rightarrow 0$ limit for coherent states. Commun. Math. Phys. 71, 77-93 (1980)

9. Hagedorn, G. A. : Semiclassical quantum mechanics II : the large mass asymptotics for coherent states. In: Trends in applications of pure mathematics to mechanics Vol. III (ed. R. J. Knops). London: Pitman Publishing Ltd. (to appear 1980)

10. Heller, E. J. : Classical S-matrix limit of wave-packet dynamics. J. Chem. Phys. 65, 4979-4989 (1976)

11. Hepp, K. : The classical limit for quantum mechanical correlation functions. Commun. Math. Phys. 35, 265-277 (1974)

12. Kato, T. : On the adiabatic theorem of quantum mechanics. J. Phys. Soc. Jpn. 5, 435-439 (1950)

13. Kato, T. : Perturbation theory for linear operators. Berlin, Heidelberg, New York : Springer 1966

14. London, F. : Über den Mechanismus der homoöpolaren Bindung. In: Probleme der modernen Physik (ed. P. Debye). Leipzig: S. Hirzel 1928

15. Nenciu, G. : On the adiabatic theorem of quantum mechanics. Preprint, Central Institute of Physics, Institute for Physics and Nuclear Engineering, Bucharest, Romania 1979

16. Reed, M., Simon, B. : Methods of modern mathematical physics, Vol. I: functional analysis. New York, London: Academic Press 1972

17. Reed, M., Simon, B.: Methods of modern mathematical physics, Vol. III : scattering theory. New York, London: Academic Press 1979

18. Reed, M., Simon, B. : Methods of modern mathematical physics, Vol. IV : analysis of operators. New York, London: Academic Press 1978

Communicated by B. Simon

Received January 29, 1980; in revised form March 25, 1980 
